\title{
A Dynamic P53-MDM2 Model with Time Delay
}

\author{
Gh. I. MIHALAŞ, M. NEAMŢU, D. OPRIŞ, F. HORHAT
}

\begin{abstract}
Specific activator and repressor transcription factors which bind to specific regulator DNA sequences, play an important role in gene activity control. Interactions between genes coding such transcription factors should explain the different stable or sometimes oscillatory gene activities characteristic for different tissues. Starting with the model P53-MDM2 described into [6] and the process described into [5] we developed a new model of this interaction. Choosing the delay as a bifurcation parameter we study the direction and stability of the bifurcating periodic solutions. Some numerical examples are finally given for justifying the theoretical results.
\end{abstract}

Keywords: delay differential equation, stability, Hopf bifurcation, P53, MDM2. 2000 AMS Mathematics Subject Classification:34K18, 34K20, 34K13, 92D10.

\section{Introduction}

It is well-known that in cancer, the tumor suppressor protein P53 and its partner MDM2 plays an important role. Responses to genotoxic stress are regulated by a complex network which center is formed by P53 and MDM2. The research effort in P53-MDM2 field is based on the fact that in cancer the defects of P53-MDM2 network are almost universal.

There are 2 types of P53 activity depending of its level: when the level is low or there are brief elevation, P53 activates the transcription of the genes leading to cell cycle delay at DNA damage-induced checkpoints; when the level is high or there are prolonged elevations, p53 activates the transcription 
of the genes leading to apoptosis. These opposite outcomes are determined, at least in part, by cofactors.

Between p53 and mdm2 exists a reciprocal relationship: p53 stimulates the transcription of the mdm2 gene, and thus increase the synthesis of MDM2 protein; on the other hand MDM2 protein, through its RING domain in the C-terminus, ubiquinates P53 and stimulates its degradation. This mechanism appears to form a negative feedback loop. We said it appears, because recent findings put a question mark on this loop. This arises from the fact that there are 2 MDM2 proteins: P90MDM2 and P76MDM2 [7]. P53 acts on the promoter P2 of the P76MDM2 and thus stimulates its production but P76MDM2 has a truncated N-terminus and can not bind P53 protein and thus can not ubiquinate P53, which is needed for degradation of P53. Instead, P90MDM2 has a complete N-terminus so it can bind P53 and thus can promote P53 degradation. For the loop to be complete, it requires an interaction between P76MDM2 and P90MDM2, more precisely P76MDM2 should stimulate P90MDM2 binding to P53 or more, P76MDM2 should act on P1 promoter of P90MDM2, directly or through an intermediary substance or as a cofactor. Because the details of the interactions of the MDM2 isoforms (P76 and P90) are only at the beginning to be worked out [7], and because the activators of the $\mathrm{P} 1$ promoter of the MDM2 (for the P90MDM2 protein) are unknown, it could be suspected a link between P76MDM2 and P90MDM2 that close the loop (in the way presented above). Till now, there are no findings to negate this link and moreover, using this loop in our model, we obtain an oscillatory behavior similar to that observed experimentally $[2,3]$.

We used as a base for our model the model described in [6]. We will present that model below:

$$
\left\{\begin{array}{l}
\dot{x}_{1}=\varphi A_{1}-\eta_{1} x_{1} \\
\dot{y}_{1}=\psi x_{1}-\left(\lambda_{1}+\lambda_{12} y_{2}\right) y_{1} \\
\dot{x}_{2}=\varphi f\left(y_{1}(t-\tau)\right)-\eta_{2} x_{2} \\
\dot{y}_{2}=\psi x_{2}-\lambda_{2} y_{2}
\end{array}\right.
$$

where: $\varphi$ is the rate for transcription (maximal) $\Psi$ is the rate for translation (maximal), $\eta_{1}, \eta_{2}$ are the rates for mRNA degradation, $\lambda_{1}, \lambda_{2}, \lambda_{12}, \lambda_{21}$ are the rates for protein degradation, $x_{1}, x_{2}$ are mRNA concentrations, $y_{1}, y_{2}$ are protein concentrations and $A_{1}$ is the maximal activation degree for the transcription of the p53-gene. 
Our model is:

$$
\left\{\begin{array}{l}
\dot{x}_{1}=\varphi A_{1}-\eta_{1} x_{1}(t) \\
\dot{y}_{1}=\psi x_{1}(t)-\left(\lambda_{1}+\lambda_{12} y_{2}\left(t-\tau_{1}\right)\right) y_{1}(t) \\
\dot{x}_{2}=\varphi f\left(y_{1}\left(t-\tau_{2}\right)\right)-\eta_{2} x_{2}(t) \\
\dot{y}_{2}=\psi x_{2}(t)-\left(\lambda_{2}+\lambda_{21} y_{1}\left(t-\tau_{3}\right)\right) y_{2}(t)
\end{array}\right.
$$

The notations are identical as the previous ones and: $\tau_{1}$ is the delay required for MDM2 to bind P53 plus the time required for the interaction (under research) between P76MDM2 - P90MDM2, and also include the time for translocation of P53 in cytosol [5] (this is also a mechanism for the downregulation of $\mathrm{P} 53) . \tau_{2}$ is the delay required for $\mathrm{P} 53$ to enter in the nucleus to bind $\mathrm{P} 2$ promoter of the mdm2 gene. $\tau_{3}$ is the delay required for the HAUSP to interact with P53 and MDM2 and to deubiquinate both proteins. $\lambda_{21}$ is degradation rate for MDM2 protein induced by P53. Recent findings show that HAUSP (also known as USP7), an ubiquitin hydrolase, plays a role in P53-MDM2 degradations. Its role, in the presence of P53, is to deubiquinate MDM2 and keeps a high MDM2 level. To simplify the expressions that will appear in the calculus we use some notations: $\eta_{1}=b_{1}, \lambda_{1}=a_{1}, \lambda_{12}=a_{12}$, $\eta_{2}=b_{2}, \lambda_{2}=a_{2}, \lambda_{21}=a_{21}$ and also put numerical values for some parameters as follows: $\varphi=1, \psi=1, A_{1}=1$. These changes have no mathematically effect on our system. Finally, we will consider $\tau_{1}=\tau_{2}=\tau_{3}=\tau$, the reason is that without this hypothesis the calculus become extremely complicated and the final result will not differ qualitatively from the calculus with this hypothesis. With these specifications made, our system became:

$$
\begin{aligned}
& \dot{x}_{1}(t)=1-b_{1} x_{1}(t), \\
& \dot{y}_{1}(t)=x_{1}(t)-\left(a_{1}+a_{12} y_{2}(t-\tau)\right) y_{1}(t), \\
& \dot{x}_{2}(t)=f\left(y_{1}(t-\tau)\right)-b_{2} x_{2}(t), \\
& \dot{y}_{2}(t)=x_{2}(t)-\left(a_{2}+a_{21} y_{1}(t-\tau)\right) y_{2}(t)
\end{aligned}
$$

where $f: \mathbb{R}_{+} \rightarrow \mathbb{R}$, is the Hill function, given by:

$$
f(x)=\frac{x^{n}}{a+x^{n}}
$$

with $n \in \mathbb{N}^{*}, a>0$. The parameters of the model are assumed to be positive numbers less or equal than 1 .

For $\tau_{1}=0, \tau_{2}=0, a_{21}=0$ in our model, we obtain the model from [6]. 
The paper is organized as follows. In section 2, local stability for the equilibrium state of system (1) is discussed. We investigate the existence of the Hopf bifurcation for system (1) using time delay as the bifurcation parameter. In section 3, the direction of Hopf bifurcation is analyzed by the normal form theory and the center manifold theorem introduced by Hassard [4]. Numerical simulations for justifying the theoretical results are illustrated in section 4. Finally, some conclusions are done and further research directions are presented.

\section{Local stability and the existence of the Hopf bifurcation.}

For the study of the model (1) we consider the following initial values:

$$
x_{1}(0)=\bar{x}_{1}, y_{1}(\theta)=\varphi_{1}(\theta), \theta \in[-\tau, 0], x_{2}(0)=\bar{x}_{2}, y_{2}(\theta)=\varphi_{2}(\theta), \theta \in[-\tau, 0] \text {, }
$$

with $\varphi_{1}, \varphi_{2}$ differentiable functions.

From (1) and (2), we have:

Proposition 1. If $y_{10}$ is one positive root of the equation:

$$
\begin{aligned}
& a_{1} b_{1} b_{2} a_{21} x^{n+2}+\left(b_{1} a_{12}-b_{2} a_{21}+a_{1} b_{1} a_{2} b_{2}\right) x^{n+1}-a_{2} b_{2} x^{n}+ \\
& +a_{1} b_{1} b_{2} a a_{21} x^{2}+\left(b_{1} a a_{2} a_{1} b_{2}-b_{2} a a_{21}\right) x-a a_{2} b_{2}=0,
\end{aligned}
$$

then the equilibrium state of system (1) is given by:

$$
x_{10}=\frac{1}{b_{1}}, \quad y_{10}, \quad x_{20}=\frac{1}{b_{1}} f\left(y_{10}\right), \quad y_{20}=\frac{f\left(y_{10}\right)}{b_{1}\left(a_{2}+a_{21} y_{10}\right)} .
$$

The proof is immediately. The system should be resolved by nullifying the right side of $(1)$.

We can notice that equation (3) has at least one positive real root. Indeed, if:

$$
\begin{aligned}
& k(x)=a_{1} b_{1} b_{2} a_{21} x^{n+2}+\left(b_{1} a_{12}-b_{2} a_{21}+a_{1} b_{1} a_{2} b_{2}\right) x^{n+1}-a_{2} b_{2} x^{n}+ \\
& +a_{1} b_{1} b_{2} a a_{21} x^{2}+\left(b_{1} a a_{2} a_{1} b_{2}-b_{2} a a_{21}\right) x-a a_{2} b_{2}=0,
\end{aligned}
$$

then

$$
k(0)=-a a_{2} b_{2}<0, \quad \lim _{t \rightarrow \infty} k(x)=\infty .
$$

So that there is at least one equilibrium point with positive components. 
We consider the following translation:

$$
x_{1}=u_{1}+x_{10}, y_{1}=u_{2}+y_{10}, x_{2}=u_{3}+x_{20}, y_{2}=u_{4}+y_{20} .
$$

With respect to (5), system (1) can be expressed as:

$$
\begin{aligned}
& \dot{u}_{1}(t)=-b_{1} u_{1}(t), \\
& \dot{u}_{2}(t)=u_{1}(t)-\left(a_{1}+a_{12} y_{20}\right) u_{2}(t)-a_{12} y_{10} u_{4}(t-\tau)-a_{12} u_{2}(t) u_{4}(t-\tau), \\
& \dot{u}_{3}(t)=f\left(u_{2}(t-\tau)+y_{10}\right)-b_{2} u_{3}(t)-b_{2} x_{20}, \\
& \dot{u}_{4}(t)=u_{3}(t)-\left(a_{2}+a_{21} y_{10}\right) u_{4}(t)-a_{21} y_{20} u_{2}(t-\tau)-a_{21} u_{2}(t-\tau) u_{4}(t) .
\end{aligned}
$$

System (6) has $(0,0,0,0)$ as equilibrium point.

To investigate the local stability of the equilibrium state we linearize system (6). We expand it in a Taylor series around the origin and neglect the terms of higher order than the first order. Letting $X(t)=\left(v_{1}(t), v_{2}(t)\right.$, $\left.v_{3}(t), v_{4}(t)\right)^{T}$ be the linearized system variables, system (6) can be expressed as:

$$
\dot{X}(t)=A X(t)+B X(t-\tau)
$$

where

$$
A=\left(\begin{array}{cccc}
-b_{1} & 0 & 0 & 0 \\
1 & -a_{1}-a_{12} y_{20} & 0 & 0 \\
0 & 0 & -b_{2} & 0 \\
0 & 0 & 1 & -a_{2}-a_{21} y_{10}
\end{array}\right), B=\left(\begin{array}{cccc}
0 & 0 & 0 & 0 \\
0 & 0 & 0 & -a_{12} y_{10} \\
0 & \rho_{1} & 0 & 0 \\
0 & -a_{21} y_{20} & 0 & 0
\end{array}\right)
$$

and $\rho_{1}=f^{\prime}\left(y_{10}\right)$.

The characteristic equation corresponding to system (7) is $\operatorname{det}(\lambda I-A-$ $\left.B e^{-\lambda \tau}\right)=0$ which leads to:

$$
\left(\lambda+b_{1}\right)\left(\lambda^{3}+b \lambda^{2}+c \lambda+d+(g \lambda+h) e^{-2 \lambda \tau}\right)=0,
$$

where

$$
\begin{aligned}
& b=a_{1}+a_{2}+b_{2}+a_{12} y_{20}+a_{21} y_{10}, \\
& c=b_{2}\left(a_{1}+a_{2}+a_{12} y_{20}+a_{21} y_{10}\right)+\left(a_{1}+a_{12} y_{20}\right)\left(a_{2}+a_{21} y_{10}\right), \\
& d=b_{2}\left(a_{1}+a_{12} y_{20}\right)\left(a_{2}+a_{21} y_{10}\right), \\
& g=-a_{12} y_{10} a_{21} y_{20}, h=-a_{12} y_{10}\left(b_{2} a_{21} y_{20}-\rho_{1}\right) .
\end{aligned}
$$


First, we consider the case without delay $\tau=0$. According to the RouthHurwitz criterion we have:

Proposition 2. When there is no delay, the equilibrium point $\left(x_{10}, y_{10}\right.$, $\left.x_{20}, y_{20}\right)$ of system (1) is locally asymptotically stable if and only if:

$$
b>0, \quad(c+g) b>d+h .
$$

In what follows, we investigate the existence of the Hopf bifurcation for system (1), using time delay as the bifurcation parameter. We are looking for the values $\tau_{c}$ such that the equilibrium point $\left(x_{10}, y_{10}, x_{20}, y_{20}\right)$ changes from local asymptotic stability to instability or vice versa. This is specific for the characteristic equation with pure imaginary solutions. Let $\lambda= \pm i \omega$ be these solutions. We assume $\omega>0$. It is sufficient to look for $\lambda=i \omega$, root of (8). We obtain:

$$
\left\{\begin{array}{l}
-b \omega^{2}+d+h \cos 2 \omega \tau+\omega g \sin 2 \omega \tau=0 \\
-\omega^{3}+c \omega+\omega g \cos 2 \omega \tau-h \sin 2 \omega \tau=0 .
\end{array}\right.
$$

By squaring and adding that it follows:

$$
\omega^{6}+\left(b^{2}-2 c\right) \omega^{4}+\omega^{2}\left(c^{2}-2 b d-g^{2}\right)+d^{2}-h^{2}=0 .
$$

Let it be $z=\omega^{2}$. Then (9) becomes:

$$
z^{3}+l_{1} z^{2}+l_{2} z+l_{3}=0
$$

where

$$
l_{1}=b^{2}-2 c, \quad l_{2}=c^{2}-2 b d-g^{2}, \quad l_{3}=d^{2}-h^{2} .
$$

Using straight calculus $l_{1}>0, l_{2}>0$. We have:

Proposition 3. Equation (9) has at least one simple positive root if and only if $l_{3}<0$.

Proof. If $l_{3} \geq 0$ equation (10) has no real positive roots. If $l_{3}<0$ then:

$$
F(z)=z^{3}+l_{1} z^{2}+l_{2} z+l_{3}
$$

has $\lim _{z \rightarrow \infty} F(z)=\infty$ and $F(0)=l_{3}<0$. In these conditions equation (9) has at least one simple positive root.

Since delay $\tau$ is finite, the characteristic equation (8) is a function of the delay. Hence the roots of equation (8) are also functions of the delay. 
Suppose that $\omega_{c}$ is the least positive simple root of (8). We will proof that there is $\tau_{c}$ such that $\omega\left(\tau_{c}\right)=\omega_{c}$. At this value of $\tau$, the trivial solution will lose the stability. Defining

$$
G(\lambda)=-\frac{g \lambda+h}{\lambda^{3}+b \lambda^{2}+c \lambda+d}
$$

from (8) result

$$
G(\lambda)=e^{2 \lambda \tau} .
$$

Thus, we need to find the value of $\omega$ such that

$$
|G(\lambda)|=1
$$

The corresponding values of $\tau$ are obtained from:

$$
\tau=\frac{1}{2 \omega}[\arg \{G(i \omega)\}+2 k \pi], \quad k=0,1,2, \ldots
$$

The critical delay, $\tau_{c}$ is the smallest positive value of $\tau$ satisfying (14). Differentiating (12) implicitly with respect to $\tau$, we obtain:

$$
\frac{d \lambda}{d \tau}=2 \frac{\lambda g+\lambda^{2} h}{e^{2 \lambda \tau}\left(3 \lambda^{2}+2 b \lambda-c\right)-2 \tau g+h-2 \tau h \lambda} .
$$

Then, it is evaluated at $\lambda=i \omega_{c}$ and $\tau=\tau_{c}$ :

$$
\left.\frac{d \lambda}{d \tau}\right|_{\lambda=i \omega_{c}, \tau=\tau_{c}}=2 \frac{\omega_{c} g L_{2}+\omega_{c}^{2} h L_{1}}{L_{1}^{2}+L_{2}^{2}}+2 i \frac{\omega_{c} g L_{1}+\omega_{c}^{2} h L_{2}}{€_{1}^{2}+L_{2}^{2}}
$$

where

$$
\begin{aligned}
& L_{1}=\left(-c-3 \omega_{c}^{2}\right) \cos 2 \omega_{c} \tau_{c}-2 b \omega_{c} \sin 2 \omega_{c} \tau_{c}-2 g \tau_{c}+h \\
& L_{2}=\left(-c-3 \omega_{c}^{2}\right) \sin 2 \omega_{c} \tau_{c}+2 b \omega_{c} \cos 2 \omega_{c} \tau_{c}-2 h \omega_{c} \tau_{c} .
\end{aligned}
$$

From the above analysis and the standard Hopf bifurcation theory, we have the following result:

Proposition 4. For $\tau=\tau_{c}$ :

$$
\left.\operatorname{Re}\left(\frac{d \lambda}{d \tau}\right)\right|_{\lambda=i \omega_{c}, \tau=\tau_{c}}=2 \frac{\omega_{c} g L_{2}+\omega_{c}^{2} h L_{1}}{L_{1}^{2}+L_{2}^{2}} \neq 0
$$

and a Hopf bifurcation occurs at the equilibrium state given by (4) as $\tau$ passes through $\tau_{c}$. 


\section{Direction and stability of the Hopf bifurcation}

In this section, we study the direction, stability and the period of the bifurcating periodic solutions. The used method is based on the normal form theory and the center manifold theorem introduced by Hassard [4].

From the previous section, we know that if $\tau=\tau_{c}$ then any root of (8) of the form

$$
\lambda(\tau)=\alpha(\tau) \pm i \omega(\tau)
$$

satisfies $\alpha\left(\tau_{c}\right)=0, \omega\left(\tau_{c}\right)=\omega_{0}$ and $\left.\frac{d \alpha(\tau)}{d \tau}\right|_{\tau=\tau_{c}} \neq 0$.

Define the space of continuous real-valued functions as $C=C\left(\left[-\tau_{c}, 0\right], \mathbb{R}^{4}\right)$. Expanding $f$ in $(6)$ in Taylor series around $(0,0,0,0)^{T}$ we can rewrite system (6) in the form:

$$
\dot{X}(t)=A X(t)+B X(t-\tau)+F(X(t), X(t-\tau))
$$

where

$$
\begin{aligned}
& F(X(t), X(t-\tau))=\left(0, F^{2}\left(u_{2}(t), u_{4}(t-\tau)\right), F^{3}\left(u_{2}(t-\tau)\right), F^{4}\left(u_{2}(t-\tau), u_{4}(t)\right)\right)^{T}, \\
& F^{2}\left(u_{2}(t), u_{4}(t-\tau)\right)=-a_{12} u_{2}(t) u_{4}(t-\tau), \\
& F^{3}\left(u_{2}(t-\tau)\right)=\frac{1}{2} \rho_{2} u_{2}(t-\tau)^{2}+\frac{1}{6} \rho_{3} u_{3}^{3}(t-\tau), \\
& F^{4}\left(u_{2}(t-\tau), u_{4}(t)\right)=-a_{21} u_{2}(t-\tau) u_{4}(t), \rho_{2}=f^{\prime \prime}\left(y_{10}\right), \rho_{3}=f^{\prime \prime \prime}\left(y_{10}\right) . \\
& \quad \text { In } \tau=\tau_{c}+\mu, \mu \in \mathbb{R}, \text { we regard } \mu \text { as the bifurcation parameter. For } \\
& \Phi \in C \text { we define a linear operator: }
\end{aligned}
$$

$$
L(\mu) \Phi=A \Phi(0)+B \Phi(-\tau)
$$

and a nonlinear operator:

$$
F(\mu, \Phi)=\left(0, F^{2}\left(\Phi_{2}(0), \Phi_{4}(-\tau)\right), F^{3}\left(\Phi_{2}(-\tau)\right), F^{4}\left(\Phi_{2}(-\tau), \Phi_{4}(0)\right)\right)^{T}
$$

For $\Phi \in C^{1}\left(\left[-\tau_{c}, 0\right], \mathbb{C}^{* 4}\right)$ we define:

$$
\mathcal{A}(\mu) \Phi(\theta)= \begin{cases}\frac{d \Phi(\theta)}{d \theta}, & \theta \in\left[-\tau_{c}, 0\right) \\ A \Phi(0)+B \Phi\left(-\tau_{c}\right), & \theta=0\end{cases}
$$




$$
R(\mu) \Phi(\theta)= \begin{cases}0, & \theta \in\left[-\tau_{c}, 0\right) \\ F(\mu, \Phi), & \theta=0\end{cases}
$$

and for $\Psi \in C^{1}\left(\left[0, \tau_{c}\right], \mathbb{C}^{* 4}\right)$, we define the adjoint operator $\mathcal{A}^{*}$ of $\mathcal{A}$ by:

$$
\mathcal{A}^{*} \Psi(s)= \begin{cases}-\frac{d \Psi(s)}{d s}, & s \in\left(0, \tau_{c}\right] \\ A \Psi(0)+B \Psi\left(\tau_{c}\right), & s=0 .\end{cases}
$$

Then, we can rewrite (16) in the following vector form:

$$
\dot{X}_{t}=A(\mu) X_{t}+R(\mu) X_{t}
$$

where $X_{t}=X(t+\theta)$ for $\theta \in\left[-\tau_{c}, 0\right]$. For $\Phi \in C^{1}\left(\left[-\tau_{c}, 0\right], \mathbb{C}^{* 4}\right)$ and $\Psi \in$ $C\left(\left[0, \tau_{c}\right], \mathbb{C}^{* 4}\right)$ we define the following bilinear form:

$$
<\Psi(\theta), \Phi(\theta)>=\Psi(0) \Phi(0)+\int_{-\tau_{c}}^{0} \Psi\left(\theta+\tau_{c}\right) B \Phi(\theta) d \theta
$$

Then, it can verified that $\mathcal{A}^{*}$ and $\mathcal{A}(0)$ are adjoint operators with respect to this bilinear form.

In the light of the obtained results in the last section, we assume that $\pm i \omega_{c}$ are eigenvalues of $\mathcal{A}(0)$. Thus, they are also eigenvalues of $\mathcal{A}^{*}$. We can easily obtain:

$$
\Phi(\theta)=v e^{\lambda_{1} \theta}, \quad \theta \in\left[-\tau_{c}, 0\right]
$$

where $v=\left(v_{1}, v_{2}, v_{3}, v_{4}\right)^{T}$,

$$
v_{1}=0, v_{2}=\frac{\lambda_{1}+b_{2}}{\rho_{1}} e^{\lambda_{1} \tau}, v_{3}=1, v_{4}=-\frac{\left(\lambda_{1}+b_{2}\right)\left(\lambda_{1}+a_{1}+a_{12} y_{20}\right)}{\rho_{1} a_{12} y_{10}} e^{2 \lambda_{1} \tau}
$$

is the eigenvector of $\mathcal{A}(0)$ corresponding to $\lambda_{1}=i \omega_{c}$ and

$$
\Psi(s)=w e^{\lambda_{2} s}, \quad s \in\left[0, \tau_{c}\right]
$$

where $w=\left(w_{1}, w_{2}, w_{3}, w_{4}\right)$,

$$
\begin{aligned}
& w_{1}=\frac{1}{\eta}, w_{2}=\frac{b_{1}+\lambda_{1}}{\eta}, w_{3}=-\frac{a_{12} y_{10} e^{-\lambda_{1} \tau}\left(b_{1}+\lambda_{1}\right)}{\left(a_{2}+a_{21} y_{10}+\lambda_{1}\right)\left(b_{2}+\lambda_{1}\right) \eta}, \\
& w_{4}=-\frac{a_{12} y_{10} e^{-\lambda_{1} \tau}\left(b_{1}+\lambda_{1}\right)}{\left(a_{2}+a_{21} y_{10}+\lambda_{1}\right) \eta},
\end{aligned}
$$




$$
\begin{aligned}
& \eta=v_{2}\left(b_{1}+\lambda_{1}\right)-\frac{a_{12} y_{10} e^{-\lambda_{1} \tau}\left(b_{1}+\lambda_{1}\right)}{\left(a_{2}+a_{21} y_{10}+\lambda_{1}\right)\left(b_{2}+\lambda_{1}\right)} v_{3}-\frac{a_{12} y_{10} e^{-\lambda_{1} \tau}\left(b_{1}+\lambda_{1}\right)}{a_{2}+\lambda_{1}+a_{21} y_{10}} v_{4}+ \\
& +\tau e^{\lambda_{1} \tau}\left[v_{2}\left(-\rho_{1} \frac{a_{12} y_{10} e^{-\lambda_{1} \tau}\left(b_{1}+\lambda_{1}\right)}{\left(a_{2}+a_{21} y_{10}+\lambda_{1}\right)\left(b_{2}+\lambda_{1}\right)}+a_{21} y_{20} \frac{a_{12} y_{10} e^{-\lambda_{1} \tau}\left(b_{1}+\lambda_{1}\right)}{a_{2}+\lambda_{1}+a_{21} y_{10}}\right)-\right. \\
& \left.-a_{12} y_{10} v_{4}\right]
\end{aligned}
$$

is the eigenvector of $\mathcal{A}^{*}$ corresponding to $\lambda_{2}=-i \omega_{c}$.

We can verify that: $<\Psi(s), \Phi(s)>=1,<\Psi(s), \bar{\Phi}(s)>=<\bar{\Psi}(s), \Phi(s)>=$ $0,<\bar{\Psi}(s), \bar{\Phi}(s)>=1$.

Using the approach of Hassard [4], we next compute the coordinates to describe the center manifold $\Omega_{0}$ at $\mu=0$. Let $X_{t}=X(t+\theta), \theta \in\left[-\tau_{c}, 0\right]$, be the solution of equation (7) when $\mu=0$ and

$$
z(t)=<\Psi, X_{t}>, \quad w(t, \theta)=X_{t}(\theta)-2 \operatorname{Re}\{z(t) \Phi(\theta)\} .
$$

On the center manifold $\Omega_{0}$, we have:

$$
w(t, \theta)=w(z(t), \bar{z}(t), \theta)
$$

where

$$
w(z, \bar{z}, \theta)=w_{20}(\theta) \frac{z^{2}}{2}+w_{11} z \bar{z}+w_{02}(\theta) \frac{z^{2}}{2}+w_{30}(\theta) \frac{z^{3}}{6}+\ldots
$$

in which $z$ and $\bar{z}$ are local coordinates for the center manifold $\Omega_{0}$ in the direction of $\Psi$ and $\bar{\Psi}$.

For solution $X_{t}$ of equation (7), since $\mu=0$, we have:

$$
\dot{z}(t)=\lambda_{1} z(t)+g(z, \bar{z})
$$

where

$$
\begin{aligned}
g(z, \bar{z}) & =\Psi(0) F(w(z, \bar{z}, 0)+\operatorname{Re}(z(t) \Phi(0)))= \\
& =g_{20} \frac{z^{2}}{2}+g_{11} z \bar{z}+g_{02} \frac{\bar{z}^{2}}{2}+g_{21} \frac{z^{2} \bar{z}}{2}+\ldots
\end{aligned}
$$

From (17), (18) and (20) we obtain:

$$
\begin{aligned}
& g_{20}=F_{20}^{2} w_{2}+F_{20}^{3} w_{3}+F_{20}^{4} w_{4}, g_{11}=F_{11}^{2} w_{2}+F_{11}^{3} w_{3}+F_{11}^{4} w_{4}, \\
& g_{02}=F_{02}^{2} w_{2}+F_{02}^{3} w_{3}+F_{02}^{4} w_{4},
\end{aligned}
$$


where

$$
\begin{aligned}
& F_{20}^{2}=-2 a_{12} v_{2} v_{4} e^{-\lambda_{1} \tau}, F_{11}^{2}=-a_{12}\left(v_{2} \overline{4}_{4} e^{-\lambda_{2} \tau}+\bar{v}_{2} v_{4} e^{-\lambda_{1} \tau}\right) \\
& F_{02}^{2}=-2 a_{12} \bar{v}_{2} \bar{v}_{4} e^{-\lambda_{2} \tau}, F_{20}^{3}=\rho_{2} v_{2}^{2} e^{-2 \lambda_{1} \tau} \\
& F_{11}^{3}=\rho_{2} v_{2} \bar{v}_{2}, F_{02}^{3}=\rho_{2} \bar{v}_{2}^{2} e^{-2 \lambda_{2} \tau} \\
& F_{20}^{4}=-2 a_{21} v_{2} v_{4} e^{-\lambda_{1} \tau}, F_{11}^{4}=-a_{21} v_{2} \bar{v}_{4} e^{-\lambda_{1} \tau}+v_{4} \bar{v}_{2} e^{-\lambda_{2} \tau} \\
& F_{02}^{4}=-2 a_{12} \bar{v}_{2} \bar{v}_{4} e^{-\lambda_{2} \tau}
\end{aligned}
$$

and

$$
g_{21}=F_{21}^{2} w_{2}+F_{21}^{3} w_{3}+F_{21}^{4} w_{4}
$$

where

$$
\begin{aligned}
& F_{21}^{2}=-a_{12}\left(2 v_{2} w_{11}^{4}(-\tau)+\bar{v}_{2} w_{20}^{4}(-\tau)+\bar{v}_{4} w_{20}^{2}(0) e^{-\lambda_{2} \tau}+2 v_{4} w_{11}^{2}(-\tau) e^{-\lambda_{1} \tau}\right) \\
& F_{21}^{3}=\rho_{2}\left(2 v_{2} w_{11}^{2}(-\tau) e^{-\lambda_{1} \tau}+\bar{v}_{2} w_{20}^{2}(-\tau) e^{-\lambda_{2} \tau}\right)+\rho_{3} v_{2}^{2} \bar{v}_{2} e^{-\lambda_{1} \tau} \\
& F_{21}^{4}=-a_{21}\left(2 v_{2} w_{11}^{4}(-\tau) e^{-\lambda_{1} \tau}+\bar{v}_{2} w_{20}^{4}(0) e^{-\lambda_{2} \tau}+\bar{v}_{4} w_{20}(-\tau)+2 v_{4} w_{11}^{2}(-\tau)\right)
\end{aligned}
$$

with

$$
\begin{aligned}
& w_{20}^{2}(-\tau)=-\frac{g_{20}}{\lambda_{1}} v_{2} e^{-\lambda_{1} \tau}-\frac{\bar{g}_{02}}{3 \lambda_{1}} \bar{v}_{2} e^{-\lambda_{2} \tau}+E_{2}^{2} e^{-2 \lambda_{1} \tau} \\
& w_{11}^{2}(-\tau)=\frac{g_{11}}{\lambda_{1}} v_{2} e^{-\lambda_{1} \tau}-\frac{\bar{g}_{11}}{\lambda_{1}} \bar{v}_{2} e^{-\lambda_{2} \tau}+E_{1}^{2} \\
& w_{20}^{4}(-\tau)=-\frac{g_{20}}{\lambda_{1}} v_{4} e^{-\lambda_{1} \tau}-\frac{g_{20}}{3 \lambda_{1}} \bar{v}_{4} e^{-\lambda_{2} \tau}+E_{2}^{4} e^{-2 \lambda_{1} \tau} \\
& w_{11}^{4}(-\tau)=\frac{g_{11}}{\lambda_{1}} v_{4} e^{-\lambda_{1} \tau}-\frac{\bar{g}_{11}}{\lambda_{1}} \bar{v}_{4} e^{-\lambda_{2} \tau}+E_{1}^{4} \\
& w_{20}^{2}(0)=-\frac{g_{20}}{\lambda_{1}} v_{2}-\frac{\bar{g}_{02}}{3 \lambda_{1}} \bar{v}_{2}+E_{2}^{2} \\
& w_{11}^{2}(0)=\frac{g_{11}}{\lambda_{1}} v_{2}-\frac{\bar{g}_{11}}{\lambda_{1}} \bar{v}_{2}+E_{1}^{2} \\
& w_{20}^{4}(0)=-\frac{g_{20}}{\lambda_{1}} v_{4}-\frac{\bar{g}_{20}}{3 \lambda_{1}} \bar{v}_{4}+E_{2}^{4} \\
& w_{11}^{4}(0)=\frac{g_{11}}{\lambda_{1}} v_{4}-\frac{\bar{g}_{11}}{\lambda_{1}} \bar{v}_{4}+E_{1}^{4},
\end{aligned}
$$

$E_{1}^{2}, E_{1}^{4}$ respectively $E_{2}^{2}, E_{2}^{4}$ are the components of the vectors:

$$
\begin{aligned}
& E_{2}=-\left(A+e^{-2 \lambda_{1} \tau_{c}} B-2 \lambda_{1} I\right)^{-1} F_{20} \\
& E_{1}=-(A+B)^{-1} F_{11},
\end{aligned}
$$

where $F_{20}=\left(0, F_{20}^{2}, F_{20}^{3}, F_{20}^{4}\right)^{T}, F_{11}=\left(0, F_{11}^{2}, F_{11}^{3}, F_{11}^{4}\right)^{T}$. 
Based on the above analysis and calculation, we can see that each $g_{i j}$ in (21), (22) is determined by the parameters and delay from system (1). Thus, we can explicitly compute the following quantities:

$$
\begin{aligned}
& C_{1}(0)=\frac{i}{2 \omega_{c}}\left(g_{20} g_{11}-2\left|g_{11}\right|^{2}-\frac{1}{3}\left|g_{02}\right|^{2}\right)+\frac{g_{21}}{2} \\
& \mu_{2}=-\frac{\operatorname{Re}\left(C_{1}(0)\right)}{\operatorname{Re} \lambda_{1}\left(\tau_{c}\right)}, T_{2}=-\frac{\operatorname{Im}\left(C_{1}(0)\right)+\mu_{2} \operatorname{Im} \lambda^{\prime}(\tau)}{\omega_{c}}, \beta_{2}=2 \operatorname{Re}\left(C_{1}(0)\right) .
\end{aligned}
$$

In summary, this leads to the following result:

Proposition 5. In formulas (23), $\mu_{2}$ determines the direction of the Hopf bifurcation: if $\mu_{2}>0(<0)$, then the Hopf bifurcation is supercritical (subcritical) and the bifurcating periodic solutions exit for $\tau>\tau_{c}\left(<\tau_{c}\right) ; \beta_{2}$ determines the stability of the bifurcating periodic solutions: the solutions are orbitally stable (unstable) if $\beta_{2}<0(>0)$; and $T_{2}$ determines the period of the bifurcating periodic solutions: the periodic increase (decrease) if $T_{2}>0(<0)$.

\section{Numerical examples.}

For the numerical simulations we use Maple 9.5. In this section, we consider system (1) with $a_{1}=a_{2}=0.13, a_{12}=0.02, a_{21}=0.02, b_{1}=0.8$, $b_{2}=0.01, a=4 ; a_{12}=a_{21}$ because there is molecular interaction between MDM2 and P53, one molecule to one molecule. Waveform plot and phase plot are obtained by the formula:

$X(t+\theta)=z(t) \Phi(\theta)+\bar{z}(t) \bar{\Phi}(\theta)+\frac{1}{2} w_{20}(\theta) z^{2}(t)+w_{11}(\theta) z(t) \bar{z}(t)+\frac{1}{2} w_{02}(\theta) \bar{z}(t)^{2}+X_{0}$,

where $z(t)$ is the solution of $(19), \Phi(\theta)$ is given by $(18), w_{20}(\theta), w_{11}(\theta), w_{02}(\theta)$ are given by $(22)$ and $X_{0}=\left(x_{10}, y_{10}, x_{20}, y_{20}\right)^{T}$ is the equilibrium state.

For $n=2$ we obtain: $x_{10}=1.2500000, y_{10}=0.72279716, y_{20}=79.96962531$, $x_{20}=11.55208766, \mu_{2}=-15.56012572, \beta_{2}=-0.00020024, T_{2}=-0.169703418$, $\omega=0.01173958, \tau=90.21567180$. Then the Hopf bifurcation is subcritical, the solutions are orbitally stable and the period of the solution is increasing. The wave plots and the phase plot are: 


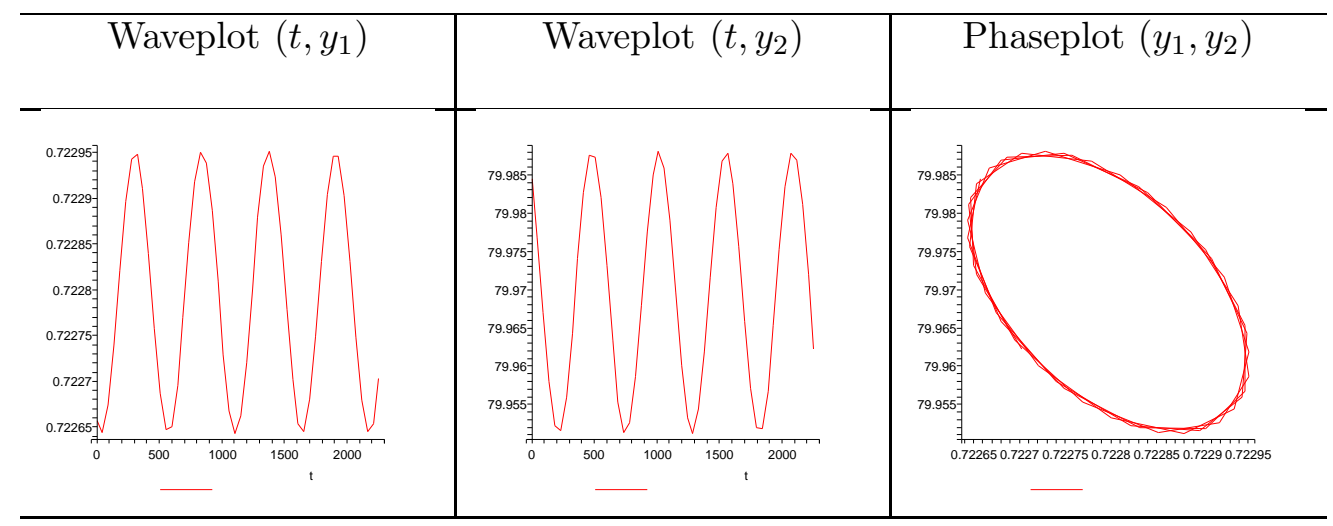

For $n=4$ we obtain: $x_{10}=1.2500000, y_{10}=0.82091152, y_{20}=69.63487984$, $x_{20}=10.19581588, \mu_{2}=-22.21740930, \beta_{2}=-0.00987558, T_{2}=-0.86252133$, $\omega=0.02969208, \tau=26.61818721$. Then the Hopf bifurcation is subcritical, the solutions are orbitally stable and the period of the solution is increasing. The wave plots and the phase plot are:

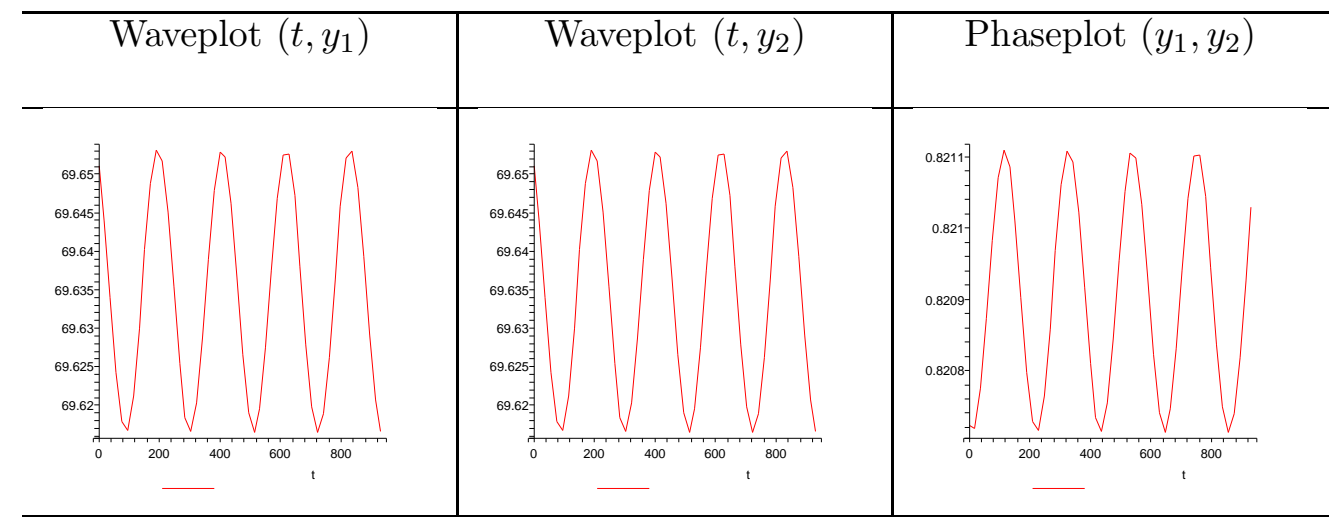

For $n=163$ we obtain: $x_{10}=1.2500000, y_{10}=0.99390609, y_{20}=56.38320475$, $x_{20}=8.45060883, \mu_{2}=-12.63855144, \beta_{2}=-0.53197047, T_{2}=7.14847952$, $\omega=0.42317766, \tau=0.00213625$. Then the Hopf bifurcation is subcritical, the solutions are orbitally stable and the period of the solution is increasing. The wave plots and the phase plot are: 


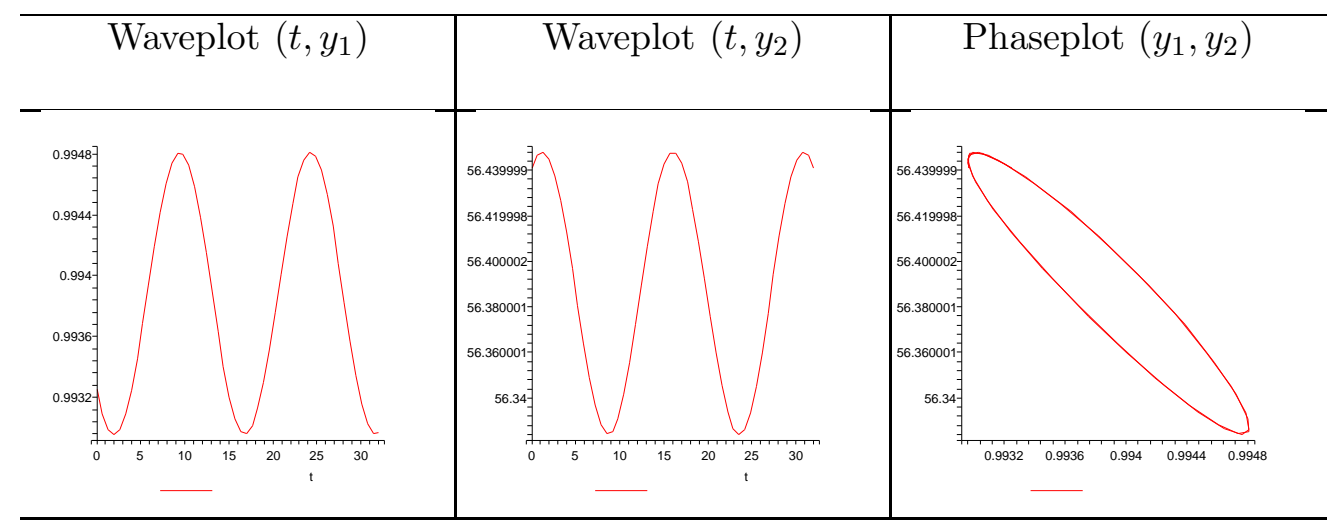

For $n=164$ we obtain: $x_{10}=1.2500000, y_{10}=0.99394289, y_{20}=56.38087608$, $x_{20}=8.45030131, \mu_{2}=4.70953378, \beta_{2}=-1.32779287, T_{2}=3.42681695$, $\omega=0.42448028, \tau=7.40096599$. Then the Hopf bifurcation is supercritical, the solutions are orbitally stable and the period of the solution is increasing. The wave plots and the phase plot are:

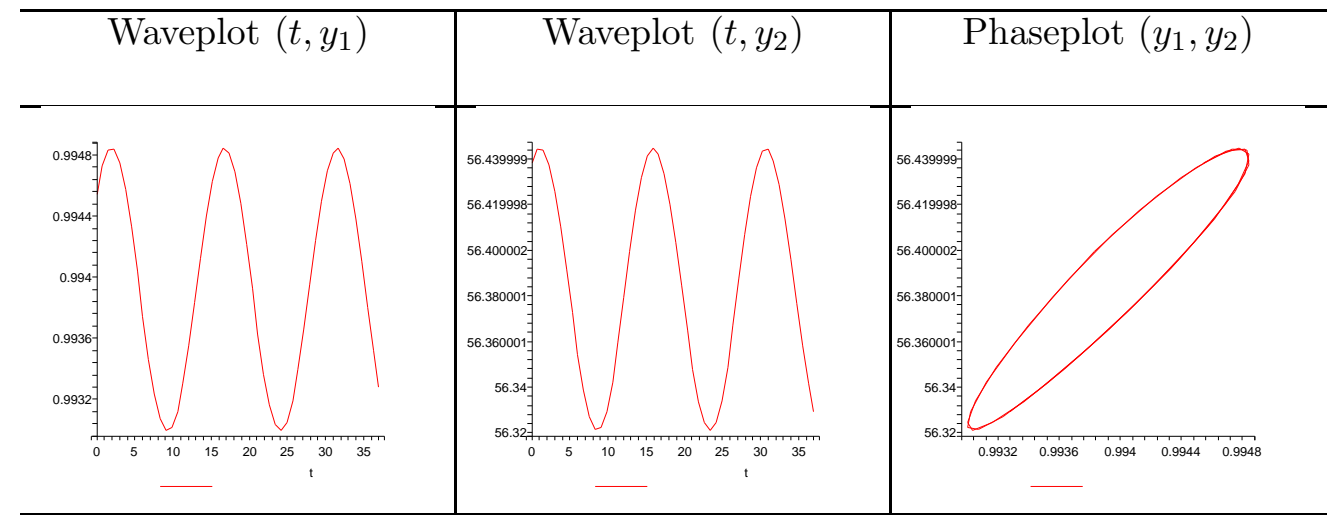

\section{Conclusions.}

Recent dynamic studies of P53 and MDM2 proteins suggest that their responses in individual cells have cyclic behavior and their characteristics are compatible with a digital clock [2]. Similar behavior we obtained in our mathematical model. The qualitative result confirms the existence of a limit cycle which can be characterized using the coefficients from (23).

The model described by (1) suggest the investigation of the model defined by the following system of nonlinear differential equation with distributed 
delay:

$$
\begin{aligned}
& \dot{x}_{1}(t)=1-b_{1} x_{1}(t), \\
& \dot{y}_{1}(t)=x_{1}(t)-\left(a_{1}+a_{12} y_{2}(t)\right) y_{1}(t), \\
& \dot{x}_{2}(t)=\frac{1}{\tau} \int_{0}^{\tau} f\left(y_{1}(t-s)\right) d s-b_{2} x_{2}(t), \\
& \dot{y}_{2}(t)=x_{2}(t)-\left(a_{2}+a_{21} y_{1}(t)\right) y_{2}(t) .
\end{aligned}
$$

The model will be studied in a future paper using the method from [1].

\section{References}

[1] M. Adimy, F. Crauste, A. Halanay, M. Neamţu, D. Opriş, Stability of Limit Cycle in a Pluripotent Stem Cell Dynamics Model, to appear in Chaos and Solitons and Fractals J. (2005)

[2] G. Lahav, N. Rosenfeld, A. Sigal, N. Geva-Zatorsky, A.J. Levine, M.B. Elowitz, U. Alon, Dynamics of the p53-Mdm2 feedback loop in individual cells, Nat. Genet. 36 (2004) 147-150.

[3] R. Lev Bar-Or, R. Maya, L.A. Segel, U. Alon, A.J.Levine, M. Oren, Generation of oscillations by p53-Mdm2 feedback loop: A theoretical and experimental study, PNAS, vol. 97, no.21 (2000), 11250-11255

[4] B.D. Hassard, N.D. Kazarinoff, Y.H. Wan, Theory and applications of Hopf bifurcation, Cambridge University Press, Cambridge, 1981.

[5] K. W. Kohn, Y. Pommier, Molecular interaction map of p53 and Mdm2 logic elements, which control the Off-On switch of $p 53$ in response to DNA damage, Science Direct, Biochemical and Biophysical Research Communications 331 (2005), 816-827

[6] G.I. Mihalas, Z. Simon, G. Balea, E. Popa, Possible oscillatory behaviour in p53-mdm2 interaction computer simulation J. of Biological Systems, vol. 8, nr. 1 (2000), 21-29

[7] M.E. Perry, Mdm2 in the response to radiation, Mol. Cancer Res. 2 (2004), 9-19 
St. I. Mihalaş

Department of Biophysics and Medical Informatics

University of Medicine and Pharmacy, Timişoara

mihalas@medinfo.umft.ro

D. Opriş

Department of Applied Mathematics

West University of Timişoara

opris@math.uvt.ro

M. Neamţu

Faculty of Economics

West University of Timişoara

mihaela.neamtu@fse.uvt.ro

F. Horhat

Department of Biophysics and Medical Informatics

University of Medicine and Pharmacy, Timişoara

rhorhat@yahoo.com 